\title{
RESULTS OF TREATMENT OF PERIPROSTHETIC FEMORAL FRACTURES AFTER TOTAL HIP ARTHROPLASTY
}

\author{
Martin Korbel, Pavel Šponer, Tomáš Kučera, Egon Procházka, Tomáš Proček
}

Charles University in Prague, Faculty of Medicine and University Hospital in Hradec Králové, Czech Republic: Department of Orthopedic Surgery

Summary: Periprosthetic fractures are the third most common reason for revision total hip arthroplasty. Surgical treatment of periprosthetic fractures belongs to the most difficult procedures due to the extensive surgery, elderly polymorbid patients and the high frequency of other complications. The aim of this study was to evaluate the results of operatively treated periprosthetic femoral fractures after total hip arthroplasty.

We evaluated 47 periprosthetic fractures in 40 patients (18 men and 22 women) operated on between January 2004 and December 2010. The mean follow-up period was 27 months (within a range of 12-45 months). For the clinical evaluation, we used modified Merle d'Aubigné scoring system.

In group of Vancouver A fractures, 3 patients were treated with a mean score of 15.7 points (good result). We recorded a mean score of 14.2 points (fair result) in 6 patients with Vancouver B1 fractures, 12.4 points (fair result) in 24 patients with Vancouver B2 fractures and 12.7 points (fair result) in 7 patients with Vancouver B3 fractures. In group of Vancouver $\mathrm{C}$ fractures, we found a mean score of 16.2 points (good result) in 7 patients.

Therapeutic algorithm based on the Vancouver classification system is, in our opinion, satisfactory. Accurate differentiation of B1 and B2 type of fractures is essential. Preoperative radiographic images may not be reliable. If in doubt, checking the stability of the prosthesis fixation during surgery should be performed.

Key words: Total hip arthroplasty; Periprosthetic femoral fracture; Vancouver classification; Complication

\section{Introduction}

Periprosthetic fractures are the third most common reason for revision total hip arthroplasty $(8,19)$. The incidence of postoperative periprosthetic femoral fractures is around $1 \%$ for primary and $4 \%$ for reimplantation (1). The most common causes of postoperative periprosthetic fractures are aseptic loosening of the arthroplasty and the patient suffering a fall. Surgical treatment of periprosthetic fractures belongs to the most difficult procedures due to the extensive surgery with increased blood loss and the high frequency of other complications (22). The treatment is often performed on elderly patients with mobility problems. Due to a reduction in both mobility and cognitive abilities, such patients often do not keep to the recommended postoperative regime. Particularly difficult to achieve is a sufficiently stable osteosynthesis. In addition, elderly patients are more at risk for of falling and injuring themselves. Johansson in his article on the treatment of periprosthetic femoral fractures demonstrated up to two thirds of cases with unsatisfactory results (11). Bhattacharyya, in a retrospective study on 106 patients with a periprosthetic fracture of the femur, revealed an $11 \%$ patient mortality. In the same study, the mortality rate in the control group after primary implantation of total prosthesis was $2.9 \%$ (3).
The aim of this retrospective study was to assess the results of operatively treated periprosthetic femoral fractures after total hip arthroplasty and evaluate the indications for surgery in our patients to improve the therapeutic approach.

\section{Material and Methods}

In a retrospective study, we focused on postoperative periprosthetic femoral fractures treated in our department from January 2004 to December 2010. The following were not included in the study group: periprosthetic acetabular fractures, intraoperative and conservatively treated femoral fractures. In total, we evaluated 47 periprosthetic fractures in 40 patients ( 18 men and 22 women). In 27 cases there was a fracture around cemented prosthesis and in 20 cases cementless (in B2 group 14 cemented and 10 cementless). The mean age of the patients was 72 years (age range 54 to 88 years). The average interval between total hip arthroplasty and surgery for fracture was 7 years and 3 months. Periprosthetic fractures were classified according to the Vancouver classification (Figure 1, Table 1) (6). When we divided the patients into the groups of the Vancouver classification, we evaluated the preoperative radiographs. As a symptom of loose femoral component we considered 
Tab. 1: Vancouver classification

\begin{tabular}{|l|l|l|l|l|l|l|}
\hline & \multicolumn{3}{|l|}{ Vancouver A } & \multicolumn{2}{l|}{ Vancouver B } & Vancouver C \\
\hline & AG & AL & B1 & B2 & B3 & C \\
\hline Prosthetic Fixation & stable & stable & stable & loose & loose & stable \\
\hline Bone Quality & good & good & good & good & poor & good \\
\hline
\end{tabular}

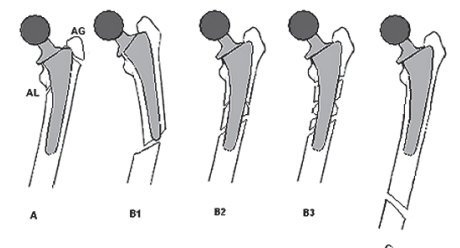

Fig. 1: Vancouver classification

radiolucent zone around the stem wider than $2 \mathrm{~mm}$ and the fracture line indicating the release of the stem. Regarding comorbidities, we focused primarily on diseases affecting patient mobility. 4 patients were post-stroke, 3 patients were being treated for Parkinson's disease, 3 patients for Bechterew's disease, 2 patients suffered from peripheral nerve paresis and one patient was being treated for alcoholism.

\section{Surgical Procedure}

The anterolateral approach to the hip was used in cases of Vancouver A or B fractures and the lateral approach to the femur was done in cases of Vancouver $\mathrm{C}$ fractures. If in doubt, whether it is a Vancouver B1 or B2 fracture, we dislocated the femoral component from the acetabulum and checked the stability of the stem. In the case of a loosened stem, we performed reimplantation of the femoral component. In the case of well fixed stem, we used plate fixation. The wound was drained by two drains and the drains were extracted in the second postoperative day. The perioperative antibiotics utilized were Cefazolin $2 \mathrm{~g}$ intravenously 30 minutes prior to incision, followed by $1 \mathrm{~g}$ after every two hours of the operation and $1 \mathrm{~g} 8$ and 16 hours after the first dose. In prevention of thromboembolism, we used low molecular weight heparin in doses according to the patient's weight. Postoperative verticalization occurred from the first up to the sixth postoperative day. The patients were monitored clinically and radiographically at 6 weeks after surgery, at 3, 6 and 12 months after surgery and then at intervals of 1 year. The mean follow-up period was 27 months (within a range of $12-45$ months). For the clinical evaluation, we used modified Merle d'Aubigné scoring system (Table 2) (16).

\section{Results}

In group of Vancouver A fractures, 3 patients were treated by cerclage fixation (Figure 2: A/B). We achieved good result with a mean score of 15.7 points according to the Merle d'Aubigné scoring system (standard devi-
Tab. 2: Modified Merle d'Aubigné scoring system

\begin{tabular}{|l|l|}
\hline Pain & Points \\
\hline none & 6 \\
\hline slight or intermittent & 5 \\
\hline after walking but resolves & 4 \\
\hline moderately severe but patient is able to walk & 3 \\
\hline severe, prevents walking & 2 \\
\hline intensive, pernament & 1 \\
\hline Walk & \\
\hline normal & 6 \\
\hline no cane but slight limp & 5 \\
\hline long distance with cane or crutches & 4 \\
\hline limited even with support & 3 \\
\hline very limited & 2 \\
\hline unable to walk & 1 \\
\hline Range of motion & \\
\hline $95-100 \%$ & 6 \\
\hline $80-94 \%$ & 5 \\
\hline $70-79 \%$ & 4 \\
\hline $60-69 \%$ & 3 \\
\hline $50-59 \%$ & 2 \\
\hline till 50\% & 1 \\
\hline
\end{tabular}

\section{Clinical grade}

$\begin{array}{ll}\text { excellent } & 18 \\ \text { good } & 15-17 \\ \text { fair } & 12-14 \\ \text { poor } & \text { till } 12\end{array}$

ation 0.578 ). In each case the fracture had healed within 3 months after the surgery. No complications were reported.

In Vancouver B1 fractures, 4 patients were treated by Locking Compression Plates with cerclage tape fixation (Figure 3: A/B) and 2 patients with plates fixation only. According to Merle d'Aubigné, we achieved a fair result with a score of 14.2 points (standard deviation 0.373 ). The complications were found in two patients. In one case the femoral stem was loosened and therefore the plate fixation was broken 9 months after surgery. In this case, the stem was well fixed during surgery, but it could be re- 
Tab. 3: Evaluation of treatment results based on the Merle d'Aubigné classification

\begin{tabular}{|l|l|l|}
\hline $\begin{array}{l}\text { Types of fracture } \\
\text { (Vancouver) }\end{array}$ & $\begin{array}{l}\text { Number of } \\
\text { patients }\end{array}$ & $\begin{array}{l}\text { Merle d'Aubigné score } \\
\text { (points) }\end{array}$ \\
\hline A & 3 & 15.7 \\
\hline B1 & 6 & 14.2 \\
\hline B2 & 24 & 12.4 \\
\hline B3 & 7 & 12.7 \\
\hline C & 7 & 16.2 \\
\hline
\end{tabular}

leased during the plate fixation around the cement mantle. This patient was subsequently treated with longer revision stem reimplantation with cerclage fixation. In one case a superficial infection occurred during the postoperative period and was successfully treated with local care and antibiotics.

In Vancouver B2 fractures, we treated 18 patients with reimplantation of a revision stem (Figure 4: A/B) and 6 patients with plate osteosynthesis. According to the Merle d'Aubigné scoring system we achieved a fair result of 12.4 points (standard deviation 0.685 ). In subgroup of 18 patients treated by revision stem reimplantation, the prosthesis was dislocated in two cases. This was successfully treated by closed reduction. Two patients suffered incomplete femoral nerve palsy with complete restoration in 3 months. The femoral stem was loosened and therefore broken in one case. The patient was successfully treated by reimplantation of a longer revision stem with cerclage tape fixation. Substantial intraoperative blood loss $(2500 \mathrm{ml})$ occurred in one case. It required blood substitution to combat the developing hemorrhagic shock. In subgroup of 6 patients treated with plate osteosynthesis, the complications were found in 3 patients. The plate osteosynthesis was broken in all 3 patients (Figure 6: A/B/C). We performed long revision stem reimplantanion in 2 cases and in one case, the fracture was fixed by plate osteosynthesis and the prosthesis was removed due to the polymorbid condition of the patient and the limited mobility expectations.

In the group of Vancouver B3 fractures, we treated 3 patients by long revision stem implantation with cerclage fixation (Figure 5: A/B), 3 patients by stem implantation with proximal femoral replacement, and 1 patient by means of prosthesis extraction. According to the Merle d'Aubigné system, we achieved a fair result of 12.7 points points (standard deviation 0.451 ). The complications included 1 superficial infection succesfully treated with local care and antibiotics. In the group of Vancouver $\mathrm{C}$ fractures we treated all seven patients with plate osteosynthesis using bicortical screw fixation. We achieved a good result with a score of 16.2 points according to the Merle d'Aubigné system (standard deviation 0.769 ). The operations took place without complications. One patient complained a persistent moderate postoperative pain.

The average Merle d'Aubigné clinical score is presented in table 3. The complications found in particular Vancouver type fractures are summarized in table 4.

\section{Discussion}

Overall health status of the patient, localization and type of the fracture and femoral stem status, whether the stem is intact or loose, should be considered, when we choose the type of the surgery. Historically various classification systems have been proposed, but none of them did reflect all the factors essential for therapeutic decision (2).

Nowadays the Vancouver classification treatment algorithm for postoperative periprosthetic fractures is generally considered satisfactory. This classification takes into account the localization of the fracture in relation to the femoral component, the fixation of the femoral component in the femur and the quality of the bone tissue (8).

Tab. 4: Complications of particular fracture types

\begin{tabular}{|c|c|c|c|c|}
\hline $\begin{array}{l}\text { Types of fracture } \\
\text { (Vancouver) }\end{array}$ & $\begin{array}{l}\text { Number of } \\
\text { patients }\end{array}$ & Treatment & $\begin{array}{l}\text { Number of } \\
\text { patients treated }\end{array}$ & Complication \\
\hline A & 3 & tension band cerclage & 3 & 0 \\
\hline \multirow{2}{*}{ B1 } & \multirow{2}{*}{6} & LCP & 4 & $1 \times$ plate dislocation, $1 \times$ superficial infection \\
\hline & & Ogden plates & 2 & 0 \\
\hline \multirow[t]{2}{*}{$\mathrm{B} 2$} & \multirow[t]{2}{*}{24} & revision stem & 18 & $\begin{array}{l}2 \times \text { prosthesis luxation, } 1 \times \text { broken fermoral } \\
\text { stem, } 1 \times \text { hemorrhagic shock, } 2 \times \text { paresis of } \\
\text { the femoral nerve, } 1 \times \text { deep infection }\end{array}$ \\
\hline & & LCP & 6 & $3 \times$ broken plate fixation \\
\hline \multirow{3}{*}{ B3 } & \multirow{3}{*}{7} & revision stem & 3 & 0 \\
\hline & & tumorous stem & 3 & 0 \\
\hline & & prosthesis extraction & 1 & $1 \times$ superficial infection \\
\hline $\mathrm{C}$ & 7 & LCP & 7 & 0 \\
\hline
\end{tabular}



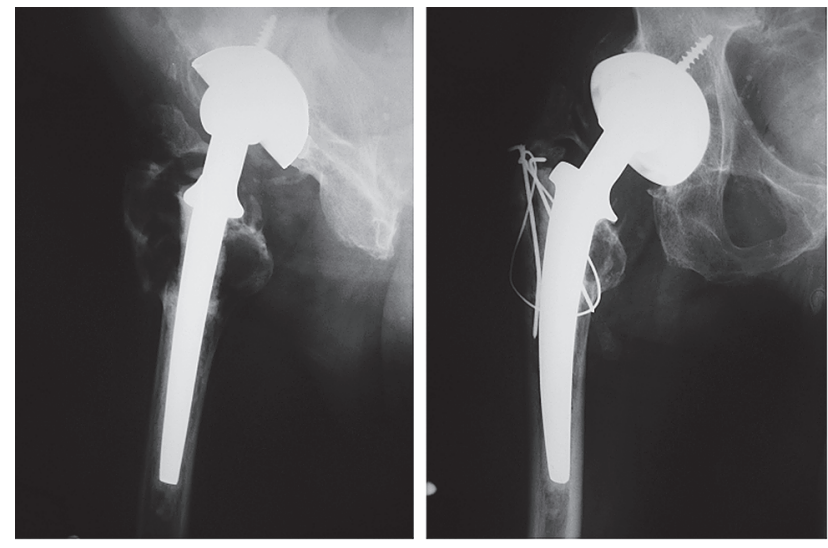

Fig. 2: A/B - A patient with a Vancouver type A fracture 11 years after primary implantation, treated with cerclage of the greater trochanter
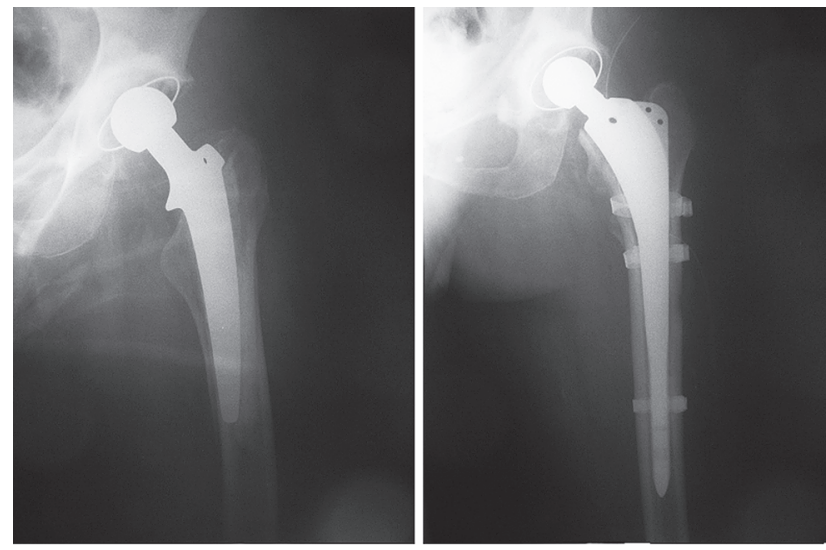

Fig. 4: A/B - A patient with a Vancouver type B2 fracture 12 years after primary implantation, treated by revision stem reimplantation and cerclage tape fixation

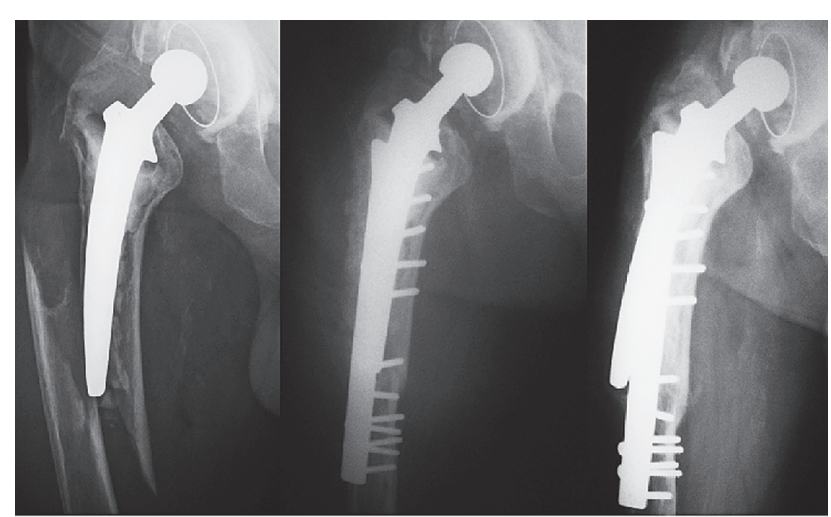

Fig. 6: A/B/C - A polymorbid patient with a Vancouver B2 fracture; due to the limited mobility expectations of the patient, plate osteosynthesis was chosen. Three months after the operation, failure of the osteosynthesis occurred
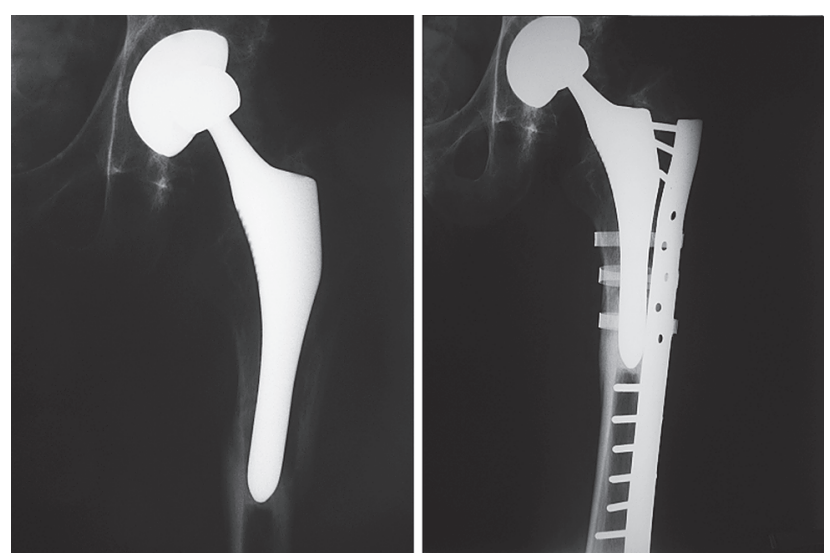

Fig. 3: A/B - A patient with a Vancouver type B1 fracture 4 years after primary implantation, treated with plate osteosynthesis
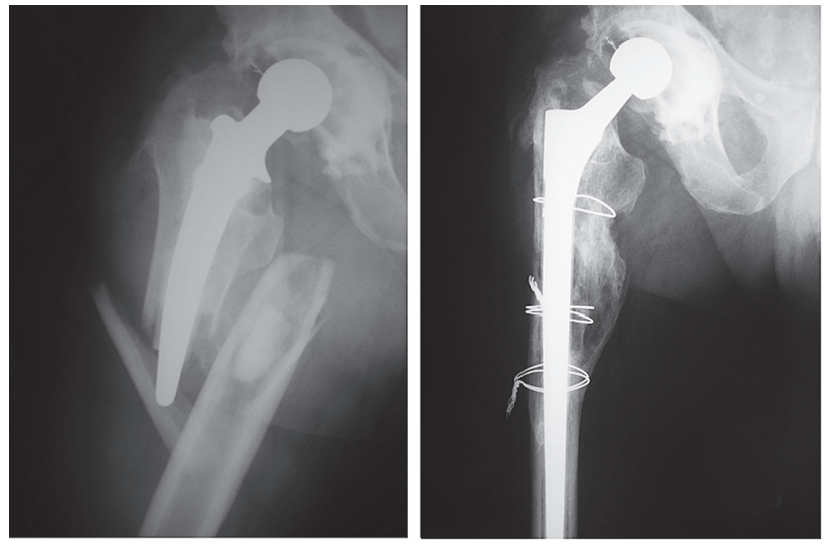

Fig. 5: A/B - A patient with a Vancouver type B3 fracture 12 years after primary implantation, treated by revision stem reimplantation and cerclage fixation

Vancouver A fractures includes both the greater and lesser trochanter. These fractures are further divided into stable and unstable types. Stable types can be treated conservatively by reducing limb weight bearing and avoidance of active abduction (10). Unstable types are treated by cerclage fixation.

Vancouver B fractures occur at the level of the femoral stem or just below its tip. In the B1 subgroup, the femoral stem is stable and surrounding bone stock is adequate. In B2 type fractures, the femoral stem is loosened while there is still good quality of the surrounding bone stock. In B3 fractures, the femoral stem is loosened with substantial bone loss. Vancouver $\mathrm{C}$ fractures are fractures of the femur distal to the stem. The important weakness of the Vancouver classification is the clinical difficulty in distinguishing between B1 and B2 fractures $(5,7,8,21)$. Lindhal reported a high percentage of periprosthetic failure in type $B$ fractures treated with plate osteosynthesis in his publications 
$(13,14,15)$. The reason given was an error in judgment by the surgeon, who mistook an unstable type of prosthesis for a stable prosthesis. Bhattacharyya (3) in a retrospective study surprisingly reported significantly lower mortality in patients with type B fractures treated with reimplantation of the femoral component with cerclage versus those treated by plate osteosynthesis. Plate osteosynthesis is, however, a less extensive type of surgery with a shorter operating time and less blood loss. Bhattacharyya claims that the possibility of faster verticalization of patients when stable stem reimplantation methods are used accounts for the lower mortality rate $(3,12,18)$. Fousek $(8,14)$ recommended performing an intraoperative stability test of the stem whenever X-rays fail to determine the type of fracture, i.e. $\mathrm{B} 1$ or $\mathrm{B} 2$. When there is doubt as to whether a fracture is $\mathrm{B} 1$ or $\mathrm{B} 2$, the longer revision stem bypassing the fracture with cerclage fixation to enhance the rotational stability should be performed. Unfortunately in earlier cases of our study group, the uncertain fractures were not considered as B2 types and were not treated with reimplantation of the revision stem and the cerclage fixation. In these 6 cases of B2 fractures, we used plate osteosynthesis. In half of cases, failure of the osteosynthesis occurred. This confirms the claim that plate osteosynthesis does not provide enough stability in cases where the prosthesis is loosened. Nowadays, when we are in doubt, we dislocate the femoral component from the acetabulum and check the stability of the.

In the case of implantation of the revision stem, we prefer to use modular cementless revision stems that have vertical stability. Cemented stems are rarely used, except in cases of severe osteoporosis. The fracture line should be anatomically reduced during the cementing to avoid cement getting into fracture line. This in turn could lead to nonunion (10).

B3 type fractures are difficult to solve. The most difficult task is to ensure sufficient anchoring components to deficient bone and good stability to the hip joint prosthesis (20). Soft tissues are often insufficient, and despite the restoration of the limb adequate stability in these cases is lacking. We prefer the long modular cementless stems with vertical stability. In elderly patients with minimal mobility expectations, the tumor endoprosthesis with a proximal femur replacement should be performed.

Vancouver $\mathrm{C}$ fractures can sometimes be treated as a standard femoral fracture using plate osteosynthesis bicortically fixed above and below the line of fracture. If the plate is in the proximal part and extends up to the stem of the cemented prosthesis, it is preferable to fix the plate with cerclage to prevent damage to the cement mantle and release of the prosthesis $(10,20)$. From a biomechanical point of view, overloading of the femur can occur due to the high sum total of force at work in the area between the rigid components of the prosthesis and the plate osteosynthesis (10). In cases such as these a stress fracture may occur at this given spot. This can be avoided by overlapping the components (10) (Fig. $6 \mathrm{~A} / \mathrm{B})$.

\section{Conclusion}

Therapeutic algorithm based on the Vancouver classification system is, in our opinion, satisfactory. Accurate differentiation of B1 and B2 type of fractures is essential. Preoperative radiographic images may not be reliable, and we always recommend checking the stability of the prosthesis fixation during surgery. When in doubt a fracture should be treated like type B2, i.e. requiring reimplantation of the stem, thus facilitating quicker postoperative verticalization of the patient. For reimplantation we prefer the modular cementless stems with vertical stability. When using a cemented prosthesis in elderly patients, it is necessary to achieve anatomical reduction of fragments during cementing to prevent the leak of cement into the fracture line, which in turn could lead to nonunion. In the case of B3 fractures, the aim should be to maintain the proximal femur and its fixation to the prosthesis.

\section{Acknowledgements}

This article was supported by the programme PRVOUK P37/04.

\section{References}

1. Berry DJ. Epidemiology of periprosthetic fractures after major joint replacement: hip and knee. Orthop Clin North Am 1999; 30: 183-90.

2. Bethea JS, Deandrade JR, Fleming LL, Linenbaum SD, Welch RB. Proximal femoral fractures following total hip arthroplasty. Clin Orthop Relat Res 1982; 170: $95-106$

3. Bhattacharyya T, Chang D, Meigs JB, Estok DM. Mortality after periprosthetic fracture of the femur. J Bone Jt Surg Am 2007; 89: 2658-62.

4. Buttaro MA, Farfalli G, Paredes nm, Comba F, Piccaluga F. Locking compression plate fixation of Vancouver type-B1 periprosthetic femoral fractures. J Bone Jt Surg Am 2007; 89: 1964-9.

5. Čech O, Džupa V. Revizní operace náhrad kyčelního kloubu. Galén, 2004

6. Duncan C, Masri B. Fractures of the femur after hip replacement. Inst Course Lect 1995; 44: 293-304.

7. Engh CA, Massin P, Suther KE. Rentgenografic assessement of the biologic fixation of porous-surfaced femoral components. Clin Orthop Relat Res 1990; 257, $107-28$.

8. Fousek J, Vašek P. Dlahová osteosyntéza u periprotetických zlomenin typu Vancouver B1 a B2. Acta Chir Orthop Traum Čech 2009; 76: 410-16.

9. Franklin J, Malchau H. Risk factors for periprosthetic femoral fracture. Injury 2007; 38: 655-60.

10. Harkess JW, Crockarell JR. Campbell's operative orthopaedics, 2008, 406-11.

11. Johansson JE, Mcbroom R, Barrington TW. Fracture of the ispilateral femur in patiens with total hip replacement. J Bone Jt Surg Am 1981; 63: 1435-42.

12. Katzler A, Ince A, Wodtke J, Loehr JF. Component exchange in treatment of periprosthetic femoral fractures. J Arthroplasty 2006; 21, 572-9.

13. Lindahl H, Malchau H, Herberts P, Garellic G. Periprosthetic femoral fractures classification and demographics of 1049 periprosthetic femoral fractures from th Swedish National Hip Arthroplasty Register. J Arthroplasty 2005; 20: 857-65.

14. Lindhal H, Garellick G, Regner H, Herberts P, Malchau H. Three hundred and twenty-one periprosthetic femoral fractures. J Bone Jt Surg Am 2006; 88: 1215-22.

15. Lindahl H, Malchau H, Oden A, Garellic G. Risk factors for failure after treatment of a periprosthetic fractures of the femur. J Bone Jt Surg Br 2006; 88: $26-30$.

16. Matta JM. Fractures of the retabulum: accuracy of reduction and clinical results in patiens managed operatively within tree weeks after injury. J Bone Jt Surgery Am 1996; 78: 1632-45.

17. McElfresh EC, Coventry MB. Femoral and pelvic fractures after total hip artroplasty. J Bone Jt Surg Am 1974; 54: 483-92.

18. Mulay S, Hassan T, Birtwistle S, Power R. Management of types B2 and B3 femoral periprosthetic fractures by a tapered, fluted, and distaly fixed stem. J Arthroplasty 2005; 20: 751-6. 
19. Oshea K, Quinlan JF, Kutty S, Mulcahy D, Brady OH. The use of uncemented extensively porous-coated femoral components in the management of Vancouve B2 and B3 periprosthetic femoral fractures. J Bone Jt Surg Br 2005; 87: 1617-21.

20. Parvizi J, Rapuri VR, Purtill JJ, Sharkey PF. Treatment protocol for proxima femoral periprosthetic fractures. J Bone Jt Surg Am 2004; 86, 8-16.

21. Ryan F, Dodd M, Haddad FS. European validation of the Vancouver classification of periprosthetic proximal femoral fractures. J Bone Jt Surg Br 2008; 90, 1576-9.
22. Tomáš T. Patient-related risk factors for infected total arthroplasty. Acta Chir Orthop Traumatol Cech 2008, 75, 451-6.

23. Wedemeyer C, Russe K, von Knoch M, Saxler G. Endostal reaction in the region surrounding the stem of a cement-free prosthesis. An early radiological sign of imminent fracture of the femoral shaft? Unfallchirurg 2007; 110: 75-77.

24. Zenni EJ, Pomeroy DL, Caudle RJ. Ogden plate and other fixations for fractures complitating femoral endoprostheses. Clin Orthop Relat Res 1988; 231: 83-90.

Received: 03/02/2013

Accepted in revised form: 01/06/2013

\section{Corresponding author:}

Martin Korbel, M.D., University Hospital in Hradec Králové, Department of Orthopedic Surgery, Sokolská 581,50005 Hradec Králové, Czech Republic; e-mail: korbemar@fnhk.cz 